\title{
FONTE SÍSMICA DE PEQUENO DIÂMETRO PARA ENSAIOS ENTRE FUROS DE SONDAGENS (“CROSSHOLE")
}

Fabio Taioli

Received September 14, 1999 / Accepted May 25, 2000

\begin{abstract}
Em aplicações ligadas à área de engenharia civil, o ensaio "crosshole" (entre furos) é o método mais utilizado para determinação das propriedades elásticas dos maciços por intermédio da medida das velocidades de propagação das ondas P e S. Por outro lado, seu uso tem sido limitado somente a grandes obras, devido principalmente, aos custos envolvidos na sua execução. Isto é devido, principalmente, à necessidade da perfuração de furos especiais para acomodar os equipamentos utilizados. Com vistas a diminuir tais custos, foi desenvolvida uma fonte sísmica, de pequeno diâmetro e de baixo custo, possível de ser utilizada nos furos de sondagem normalmente executados para os ensaios SPT (Standard Penetration Test), durante a fase de investigação em solos. O diâmetro externo máximo desta fonte é de $40 \mathrm{~mm}$, o que permite instalá-la em revestimentos com $50 \mathrm{~mm}$ de diâmetro externo (46 mm interno). Dessa forma, o revestimento pode ser facilmente instalado em furo com $75 \mathrm{~mm}$ (3") de diâmetro máximo, permitindo, também, o preenchimento do espaço anelar com calda de cimento. A fonte sísmica é fixada na parede do revestimento por meio de um sistema pneumático e, por meio de um êmbolo deslizante gera principalmente ondas S. O uso dessa fonte em diversas obras tem evidenciado sua praticidade devida mormente ao seu pequeno tamanho e baixo peso.
\end{abstract}

Palavras-chave: Fonte sísmica; Ensaio entre furos; Estudo geotécnico.

SMALL SIZE SHEAR WAVE SOURCE FOR CROSSHOLE TESTS - In civil engineering applications, the crosshole test is the most utilized method to determine the propagation velocities of in situ $P$ and $S$ waves of the different strata and, consequently, to calculate the dynamic elastic parameters of rock or soil samples. Otherwise, its use has been limited just to very important studies since the associated cost is very high for smaller constructions such as residential and ordinary buildings. This is mainly due to the fact that the equipment normally used to perform these tests needs special boreholes, with diameter uncommon in the investigation phase. In order to decrease the crosshole overall cost, a simple and inexpensive seismic source was developed. This source can be used in the same boreholes made during the standard penetration test (SPT). Its maximum external diameter is $40 \mathrm{~mm}$, fitting in a PVC casing of $50 \mathrm{~mm}$ external diameter (46 mm internal). At the same time, the casing can be easily installed in a $75 \mathrm{~mm} \mathrm{(3")} \mathrm{maximum} \mathrm{diameter,} \mathrm{leaving} \mathrm{place} \mathrm{to} \mathrm{fill} \mathrm{the}$ annular space with mortar. The seismic source clamps on the borehole wall by a pneumatic system and, by means of a sliding piston, generates mainly S type waves. It has proven to be easy to use besides being lightweight and of small size.

Key words: Seismic source; Crosshole; Geotechnical study.

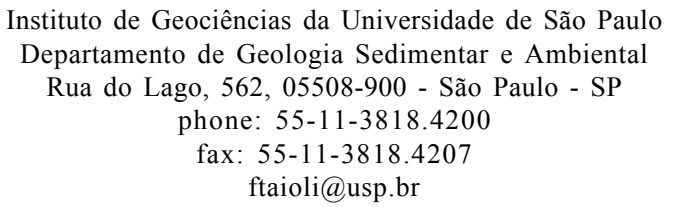




\section{INTRODUÇÃO}

O ensaio entre furos, conhecido como "crosshole", é uma aplicação do método sísmico para a determinação das velocidades de propagação das ondas sísmicas compressionais $(\mathrm{P})$ e cisalhantes $(\mathrm{S})$ nas diferentes camadas do terreno, e, conseqüentemente, para a determinação dos parâmetros elásticos dinâmicos do maciço. Esta técnica consiste na geração de ondas longitudinais e cisalhantes em um furo de sondagem no maciço e o registro da onda transmitida diretamente pela camada até um ou mais furos de sondagem paralelos ao primeiro. Desta forma, se obtém uma medida direta da velocidade de propagação das ondas $\mathrm{P}$ e S "in situ", para as condições naturais de umidade e confinamento.

Este método tem sido utilizado em obras de engenharia civil, uma vez que determina o valor do módulo de rigidez G "in situ”, amostrando um volume razoável do maciço, aplicando baixíssima deformação ao maciço, em contraposição aos ensaios estáticos.

Uma limitação que tem sido apontada como justificativa para sua pequena utilização em obras de engenharia de médio porte (e.g. edifícios, plantas industriais, pequenas barragens, etc.) é o custo considerado elevado para tal ensaio, uma vez que, com os instrumentos usuais, exige a perfuração de furos de sondagem de diâmetro especial, onerando, conseqüentemente, o custo final da obra. Este trabalho apresenta o projeto e resultados obtidos com uma fonte sísmica capaz de gerar ondas $\mathrm{P}$ e $\mathrm{S}$ em furos de sondagem com diâmetro convencionalmente utilizados em obras civis nas fases de investigação dos maciços (ensaios SPT). Testes em situações de campo têm mostrado que, sua operação em conjunto com sensor triortogonal, também de pequeno diâmetro, tem atendido às necessidades em termos de energia e discriminação entre os tipos de ondas.

\section{DETERMINAÇÃO DE CONSTANTES ELÁSTICAS INSITU}

A determinação in situ das constantes elásticas dinâmicas dos materiais naturais (solos e rochas) tem sido efetuada por meio de três métodos distintos. São eles o ensaio entre furos ("crosshole") (Hoar \&
Stokoe, 1978), a análise espectral das ondas superficiais (SASW - spectral analysis of surface waves) (Gucunski \& Woods, 1991; Stokoe II et al., 1994) ou pela sísmica de refração utilizando geofones longitudinais e transversais (Borm, 1978). A diferença básica entre o primeiro e os outros dois métodos, é que aquele é efetuado diretamente no estrato de interesse, minimizando a atenuação das ondas sísmicas e eventual dispersão. Tem sido apontado na literatura (Rector III, 1995) que a utilização de ensaios entre furos deverá sofrer um incremento, visto a diminuição progressiva nos preços dos equipamentos de registro com aumento em sua resolução, e que, efetuando ensaios em subsuperfície, são registradas freqüências mais elevadas, também contribuindo para o incremento da resolução.

O ensaio "crosshole" consiste na geração de ondas longitudinais e cisalhantes em um furo de sondagem no maciço e o registro da onda transmitida diretamente pela camada até um ou mais furos de sondagem paralelos ao primeiro. Portanto, a execução do ensaio "crosshole" envolve dois ou mais furos de sondagem revestidos com tubos de PVC, sendo que o espaço anelar entre o furo e o revestimento deve ser preenchido com calda de cimento para evitar pequenos desmoronamentos e garantir um acoplamento mecânico perfeito entre o instrumento de medida e o maciço.

A distância entre os furos deve ser planejada em função da geologia local e do horizonte a ser investigado, visto que se deve garantir que a primeira onda a chegar nos sensores seja a onda direta e não alguma onda refratada nas camadas sobre ou subjacentes. A prática tem mostrado que, em solos, se deve evitar distâncias maiores do que 5 (cinco) metros entre furos. Desta forma, esta é a distância máxima sugerida pela norma da ASTM (1990) onde o procedimento metodológico é descrito com grande detalhe. Em maciços rochosos, no entanto, esta distância pode ser maior, pois os tempos de propagação das ondas são menores. Porém, devese sempre tomar cuidado para que ondas refratadas de outros horizontes não cheguem antes do que as ondas de interesse. (Butler \& Curro, 1981).

Detalhes metodológicos podem ser encontrados também em vasta literatura, por exemplo, Stokoe \& Woods (1972), Borm (1978), Hoar \& Stokoe (1978), 
McLamore et al. (1978), Butler \& Curro (1981), Dourado (1984), Sobral et al. (1993), Prado (1994), Barros (1997), entre outros.

As velocidades de propagação das ondas elásticas são relacionadas aos módulos dinâmicos pelas seguintes equações:

$$
\begin{gathered}
V_{p}=\sqrt{\frac{E(1-v)}{\delta(1-2 v)(1+v)}} \\
V_{s}=\sqrt{\frac{E}{2 \delta(1+v)}}
\end{gathered}
$$

ou:

$$
\mathrm{V}_{\mathrm{s}}=\sqrt{\frac{\mathrm{G}}{\delta}}
$$

ou ainda:

$$
\begin{gathered}
\mathrm{G}=\mathrm{dV}_{\mathrm{s}}^{2} \\
\mathrm{v}=\frac{\left(\frac{\mathrm{V}_{\mathrm{p}}}{\mathrm{V}_{\mathrm{s}}}\right)^{2}-2}{2\left(\frac{\mathrm{V}_{\mathrm{p}}}{\mathrm{V}_{\mathrm{s}}}\right)^{2}-2}
\end{gathered}
$$

portanto,

$$
E=V_{p}{ }^{2} \delta \frac{(1-2 v)(1+v)}{(1-v)}
$$

onde:

$\mathrm{V}_{\mathrm{p}}=$ velocidade de propagação da onda $\mathrm{P}(\mathrm{m} / \mathrm{s})$;

$\mathrm{V}_{\mathrm{s}}^{\mathrm{p}}=$ velocidade de propagação da onda $\mathrm{S}(\mathrm{m} / \mathrm{s})$;

$\mathrm{d}=$ densidade do material $\left(\mathrm{kg} / \mathrm{m}^{3}\right)$;

$\mathrm{u}=$ coeficiente de Poisson dinâmico (adimensional);

$\mathrm{E}=$ módulo de elasticidade ou módulo de Young $(\mathrm{Pa})$;

$\mathrm{G}=$ módulo de rigidez $(\mathrm{Pa})$.

Outro ensaio entre furos que vem sendo utilizado no estudo de maciços é a técnica de reconstrução tomográfica. Valendo-se do desenvolvimento que esta técnica vem tendo na área médica, e sua própria utilização na indústria de óleo e gás para avaliação das características petrofísicas das rochas, a Geologia de Engenharia tem utilizado algoritmos semelhantes para resolver diversos problemas, entre eles, posicionamento espacial de fraturas, variações de qualidade do maciço ao longo do tempo, detalhamento do mapeamento geológico em zonas críticas, detecção de zonas anômalas, etc.

O princípio da tomografia sísmica é a definição da variação da velocidade de propagação de ondas sísmicas no maciço. Esta variação é função das características do maciço, uma vez que estas estão relacionadas às propriedades elásticas dos materiais e suas respectivas densidades, incluindo-se em tais propriedades as eventuais descontinuidades. $\mathrm{Na}$ prática, procura-se cobrir com o maior número de raios possível um plano do maciço, visando a obtenção de uma imagem bidimensional da variação da velocidade de propagação das ondas. Esta imagem é conseguida através do processamento adequado, baseado na discretização do plano em células, nas quais as propriedades físicas são assumidas como constantes.

Ambas aplicações do método sísmico envolvem um custo de execução que compreende, além da execução dos furos propriamente dita, também a instalação de revestimentos e preenchimento do espaço anelar (entre o revestimento e a parede do furo) com argamassa, para garantir o acoplamento sísmico entre a fonte de ondas e geofone com o maciço. Isto tem limitado, em muitos casos, a aplicação destes ensaios somente em obras de engenharia de grande porte, sendo raras as aplicações em obras mais corriqueiras.

Existem diversos sistemas instrumentais para a execução destes ensaios, principalmente para fins de detalhamento de campos petrolíferos (e.g. Elbring et al., 1989), para estudo de depósitos de rejeitos radiativos (e.g. Jung et al., 1991) ou mesmo para fins de engenharia (e.g. Sassa, 1988). No entanto, os equipamentos convencionais são construídos para serem utilizados em furos de sondagem com diâmetro mínimo de $100 \mathrm{~mm}$ (4") de forma a permitirem a instalação de revestimentos de PVC com 75 mm (3") de diâmetro. Isto requer além de furos especiais, o uso de tubos de PVC de relativos grande diâmetro e 
espessura da parede, onerando e limitando a aplicação do ensaio.

A composição do custo do ensaio "crosshole" é dada pela execução dos furos de sondagem, a instalação do revestimento, a injeção de calda de cimento no espaço anelar (entre o maciço e o revestimento), além da realização do ensaio propriamente dito, processamento dos dados e interpretação dos resultados.

Em contrapartida, a investigação geotécnica mais utilizada em solos é por meio de furos de sondagem de reconhecimento, acompanhados de ensaios de penetração (SPT - Standard Penetration Test). Estas sondagens são executadas por sistema percussivo, normalmente manual, sendo que são coletadas amostras, a cada $100 \mathrm{~cm}$, por meio do amostrador SPT para reconhecimento do material perfurado. Estes furos de sondagem, que possuem um diâmetro nominal de $75 \mathrm{~mm}$ (3 polegadas) após completados, são abandonados.

\section{SISTEMA DE ENSAIOS “CROSSHOLE”}

O equipamento para ensaios "crosshole" consiste em uma fonte sísmica, um sensor e o equipamento de registro. A fonte sísmica deve ser capaz de gerar preferencialmente ondas $S$, uma vez que a velocidade de propagação destas ondas é o parâmetro mais importante para a engenharia de fundações. No entanto, em cotas acima do nível freático, a determinação das velocidades das ondas P é também desejável para o cálculo tanto do módulo de Young como do coeficiente de Poisson dos materiais. O sensor deve ser, também, sensível a ambas as ondas, sendo que a melhor alternativa consiste no sensor que responde triortogonalmente, ou seja, sua componente vertical sendo mais sensível às ondas $\mathrm{S}$, enquanto as componentes horizontais às ondas $\mathrm{P}$, visto que as fontes geram preferencialmente ondas $\mathrm{SV}$ (ondas $\mathrm{S}$ polarizadas verticalmente).

\section{FONTE SÍSMICA}

$\mathrm{O}$ fato que norteou o desenvolvimento da fonte sísmica de pequeno diâmetro foi a necessidade de se adequar os ensaios geofísicos às práticas da engenharia geotécnica, objetivando diminuir os custos dos ensaios entre furos. Ou seja, desenvolveu-se uma fonte sísmica de pequeno diâmetro, capaz de gerar ondas $\mathrm{Pe} \mathrm{S}$, para ensaios entre furos para aplicação geotécnica. Esta fonte foi projetada e desenvolvida para ser instalada em furos de sondagem de $75 \mathrm{~mm}$ de diâmetro (3 polegadas), com revestimento de tubo de PVC de diâmetro externo de $50 \mathrm{~mm}$. Paralelamente foi desenvolvido transdutor triortogonal com características mecânicas semelhantes (Taioli, 1999), de forma a adequar ambos (fonte e transdutor) às necessidades da comunidade geotécnica.

É possível encontrar no mercado tubos de PVC com paredes de diferentes espessuras, dependendo da aplicação prevista. Desta forma, se a aplicação é para a condução de água, os tubos têm espessura compatível com a pressão a que estará submetido (cerca de $1 \mathrm{MPa}$ ). Por outro lado, os tubos destinados ao transporte de esgoto têm espessura significativamente menor pois não é esperado o acúmulo de pressão. Evidentemente, o custo destes tubos é diferenciado e proporcional à quantidade de material utilizado em sua confecção, ou seja, os tubos destinados a esgoto são significativamente menos onerosos do que aqueles destinados à água.

O desenvolvimento do sistema aqui apresentado foi efetuado levando-se em conta o maior diâmetro de tubo de PVC disponível no mercado, possível de ser colocado como revestimento em furo de sondagem de $75 \mathrm{~mm}$ (3 polegadas) de diâmetro.

Como ressaltado, tal premissa foi adotada objetivando minimizar o custo final de execução do furo de sondagem (que inclui além do furo propriamente dito, o revestimento de PVC). Em adição, apesar das sondagens a percussão com amostragem SPT serem executadas em furos de 2 $1 / 2$ " $(63,5 \mathrm{~mm})$, é prática comum efetuar o furo com 3" (75 mm) e só a amostragem no diâmetro nominal do amostrador. Portanto, pesquisa de mercado efetuada apontou que a melhor alternativa seria utilizar, como revestimento, tubos de esgoto de PVC com diâmetro externo de $50 \mathrm{~mm}$, facilmente encontrados nas lojas de materiais de construção como " $P V C$ branco $D N 50$ ". Este tubo, além de ser de baixo custo, permite um espaço anelar suficiente para que seja injetada calda de cimento que irá proporcionar um perfeito acoplamento mecânico entre a fonte sísmica e o maciço, condição necessária para a 
execução do ensaio. A junção entre os tubos é feita por cola de $\mathrm{PVC}$, que oferece grande resistência à tração e rápida aderência.

Um ponto a ser considerado é a resistência do tubo à pressão exercida pela calda de cimento, que pode ser elevada em profundidades maiores e ocasionar a deformação do tubo, obstruindo, desta forma, a passagem do equipamento. Contudo, diversos testes realizados pelo autor em ensaios vinculados a obras do Metro de São Paulo e para estudos de fundações de edifícios mostraram que, para as profundidades usuais em que os ensaios são realizados (até 35 metros), tal problema não ocorre.

A parede desse tubo de PVC tem 3,5 mm de espessura, o que o confere diâmetro interno de 43 $\mathrm{mm}$, dimensão tomada como diâmetro limite para o diâmetro externo tanto da fonte como do sensor.

A fonte sísmica apresentada neste trabalho resulta de uma evolução de fonte anteriormente desenvolvida pelo autor no Instituto de Pesquisas Tecnológicas do Estado de São Paulo - IPT, e utilizada em alguns trabalhos realizados para a Companhia do Metropolitano de São Paulo METRO. Ela é composta basicamente por três sistemas integrados, sendo um mecânico, outro elétrico e o outro pneumático. A figura 1 mostra uma perspectiva da fonte sísmica completa, pronta para ser inserida no furo de sondagem.

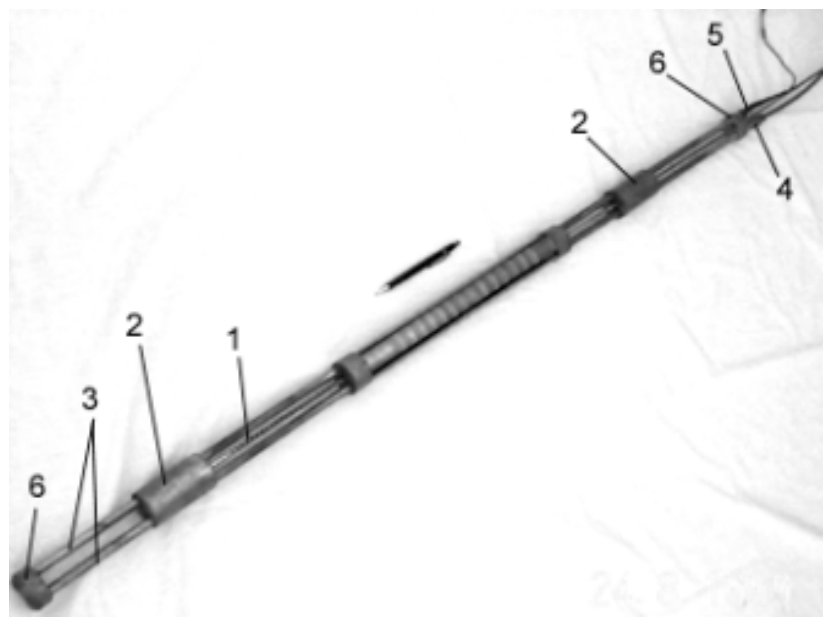

Fig. 1 - Perspectiva mostrando a fonte sísmica totalmente montada. As partes são o êmbolo deslizante (1), batedores superior e inferior (2), tubos guias (3), conexões elétrica (4) e pneumática (5) e os cilindros de alinhamento (6).

Fig. 1 - Perspective of the seismic source totally mounted. The parts are the moving piston (1), superior and inferior shockers (2), guide tubes (3), electric (4) and pneumatic (5) connections and alignment cylinders (6).

\section{Sistema mecânico}

O sistema mecânico consiste em um êmbolo deslizante com dois batedores, um em cada extremidade, que deslizam por dois tubos guias à medida que se movimenta, da superfície, um cabo de aço ao qual é preso o êmbolo. Estes batedores se chocam contra o corpo da fonte (que é fixado no maciço por meio de sistema pneumático) tanto no movimento ascendente (puxando-se o cabo de aço), como descendente (soltando-se o cabo de aço).

No movimento ascendente, o êmbolo inferior se choca com o batente inferior, enquanto no movimento descendente, o êmbolo superior se choca com o batente superior. Com isso torna-se possível gerar frentes de ondas cisalhantes com polaridades invertidas, que, por sua vez, irão gerar sinais sísmicos com polaridade invertida. A utilização desta propriedade visa facilitar a interpretação dos resultados dos ensaios, principalmente em áreas com índice de ruído ambiental elevado (caso típico de áreas urbanas), pois se torna possível determinar com grande precisão o exato instante de mudança de polaridade do sinal.

No batente superior está instalado o sistema de alimentação pneumática para que o tubo de borracha interno se infle, pressionando as sapatas contra a parede do furo. A rigidez e o alinhamento do sistema são garantidos pelos tubos guia, juntamente com dois cilindros colocados nas extremidades dos tubos guia e por um tubo interno, que une os dois batentes.

\section{Sistema elétrico}

O sistema elétrico objetiva a geração de um sinal de sincronismo ("trigger"), que indica o momento do impacto. É feito por intermédio de uma cerâmica piezoelétrica instalada em cavidade apropriada também no batente superior do corpo da fonte, sendo que o sinal gerado é transmitido por cabo elétrico blindado ao sismógrafo, a partir da extremidade de um dos tubos guia (Fig. 2).

A conexão elétrica da cerâmica piezoelétrica é feita pela carcaça do equipamento (polaridade negativa) e por meio de soldagem de um fio em sua parte superior (polaridade positiva da pastilha piezoelétrica). Um cabo blindado é utilizado para essa 


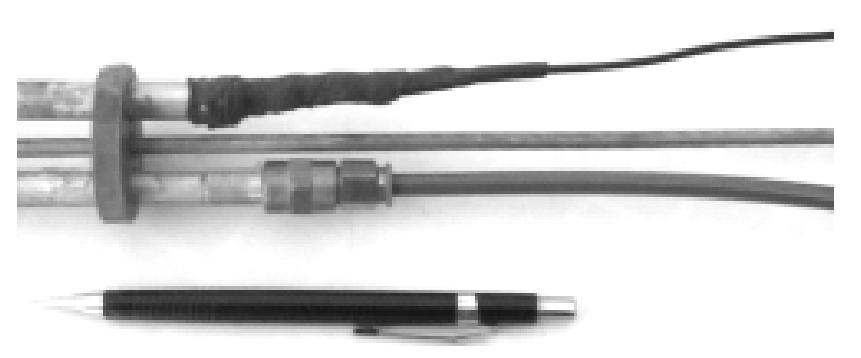

Fig. 2 - Detalhe das conexões elétrica e pneumática nas extremidades dos tubos guia.

Fig. 2 - Detail of the electrical and pneumatic connections at the ends of the guide bars.

finalidade, sendo que o condutor central transmite o sinal de polaridade positiva e a malha o sinal negativo. A malha do cabo é ligada à carcaça do equipamento por meio de um pequeno parafuso.

A cerâmica piezoelétrica, de zirconato titanato de chumbo e bário, consiste em um pequeno cilindro de $10 \mathrm{~mm}$ de diâmetro por $4 \mathrm{~mm}$ de espessura, sendo que as superfícies polarizadas são cobertas por uma película de prata condutora. Deve ser lembrado que o sinal gerado pela cerâmica piezoelétrica, apesar de ser de alta tensão (pode atingir milhares de Volts), é de baixa potência, sendo atenuado em seu percurso devido à capacitância do cabo elétrico. No entanto, este sistema mostrou-se apropriado para transmissão do sinal do "trigger" até comprimentos de cabo da ordem de 50 metros, suficiente para a grande maioria das medições de interesse em obras de engenharia (normalmente até 30 metros). O cabo elétrico, ligado à cerâmica piezoelétrica, passa pelo interior de um dos tubos guia, estando, desta forma, protegido na região de maior solicitação mecânica (região de impacto do batedor superior).

\section{Sistema pneumático}

O sistema de fixação do corpo da fonte nas paredes do furo é formado por quatro sapatas metálicas deslizantes e semiflexíveis que são pressionadas contra a parede do furo por meio de uma mangueira de borracha com alma de lona de alta resistência. Esta mangueira é inflada por ar comprimido enviado da superfície por mangueira plástica especial, de pequeno diâmetro, e conectores de engate rápido.

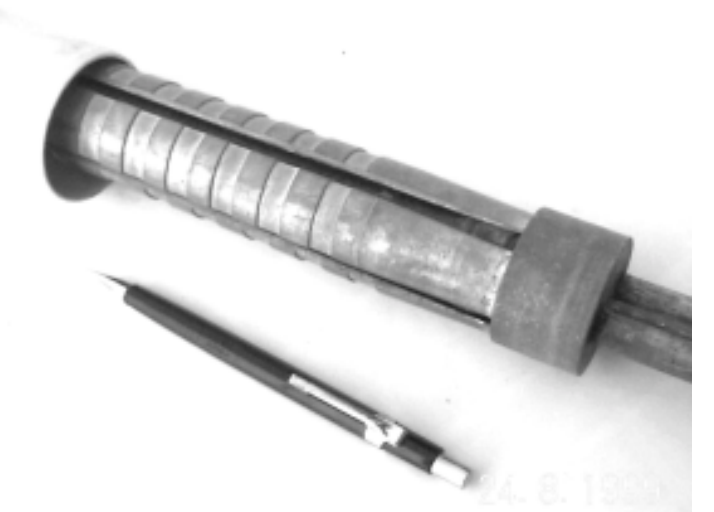

Fig. 3 - Detalhe do sistema pneumático desinflado, para instalação da fonte no furo de sondagem.

Fig. 3 - Pneumatic system depressurized, to install the source in the borehole.

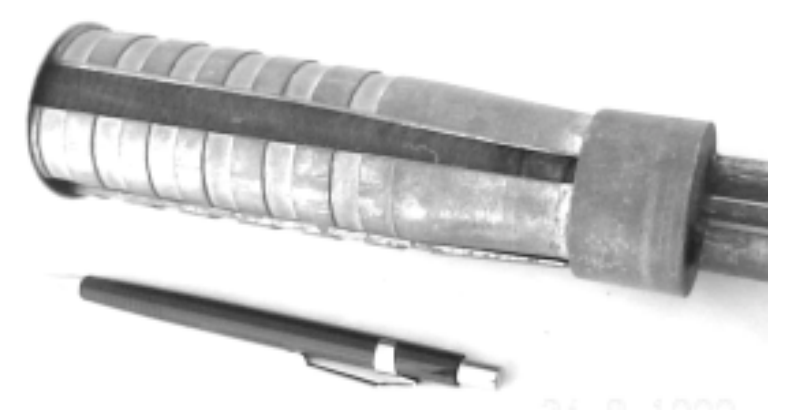

Fig. 4 - Detalhe do sistema pneumático inflado, para fixação da fonte na cota de ensaio.

Fig. 4-Pneumatic system pressurized, to clamp the source at the measurement altitude.

A entrada de ar comprimido ao sistema central (pneumático) da fonte é feita através do outro tubo guia (Fig. 2), evitando, novamente conexão direta na região de maior solicitação mecânica. Do batente superior à câmara pneumática interna, o ar comprimido é transmitido através de um orifício com cerca de $2 \mathrm{~mm}$ de diâmetro. A estanqueidade entre a mangueira de borracha e a câmara pneumática é feita por meio de uma braçadeira executada manualmente com fio ínvar (arame de aço inoxidável de alta resistência). As figuras 3 e 4 mostram respectivamente o sistema desinflado para instalação no furo de sondagem e inflado para sua fixação na cota de ensaio.

\section{TESTES REALIZADOS}

As características esperadas de uma fonte sísmica dizem respeito à quantidade de energia gerada, o que 
possibilitará o registro do sinal com uma amplitude razoável para a instrumentação disponível e seu realce sobre o ruído ambiental. A grande dúvida que existia era se as ondas sísmicas geradas teriam energia suficiente para chegar ao geofone (localizado em outro furo paralelo) com amplitude suficiente para permitir a perfeita interpretação. A pequena energia fornecida pela fonte é devida principalmente às suas pequenas dimensões (pouca massa dos batedores deslizantes e pequena altura de queda). No entanto, deve-se lembrar que os sistemas sismográficos atuais permitem que o sinal captado pelo sensor seja "empilhado" ("stacking") o que minimiza tal limitação.

Deve ser notado também que, de acordo com a norma ASTM, a distância entre os furos é especificada para ser menor ou igual a 5 metros, e mesmo com as

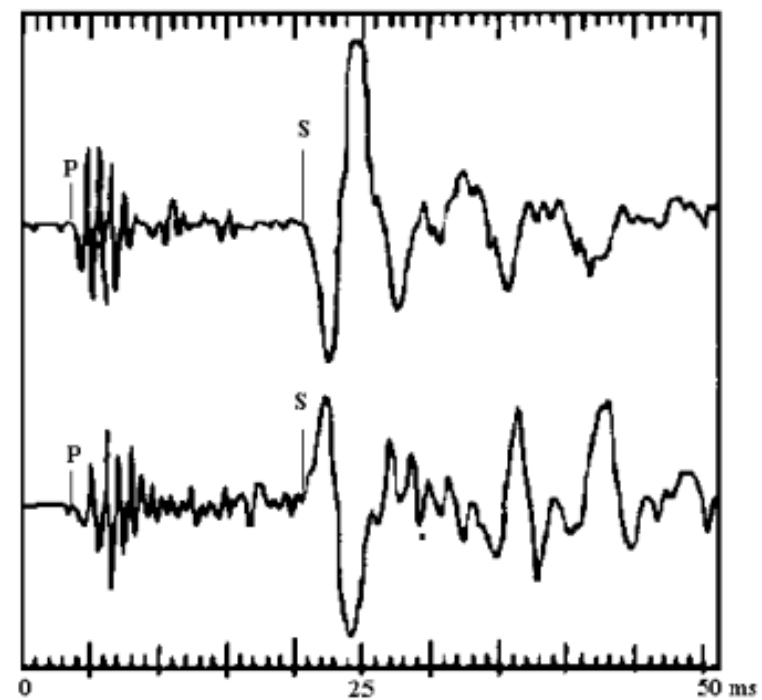

Fig. 5 - Registro mostrando a inversão da polaridade da onda S, o que possibilita sua precisa definição e medição do tempo de chegada.

Fig. 5 - Record showing polarity inversion of the $S$ wave, allowing its precise definition and measurement of the arrival time.

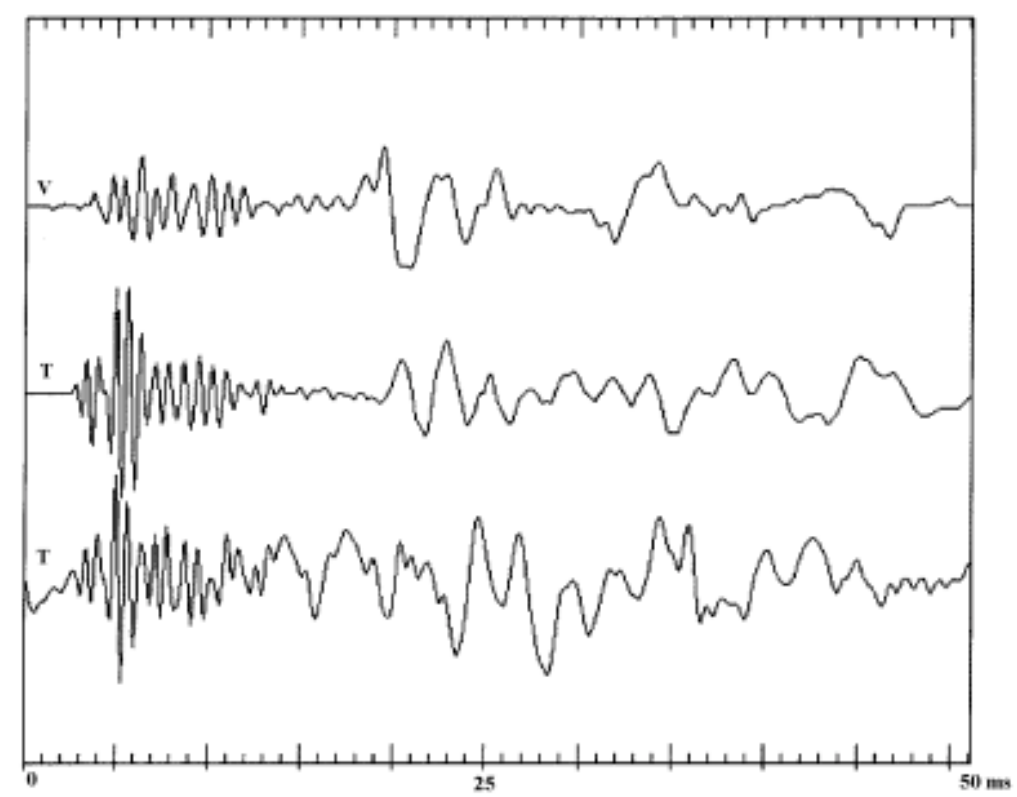

Fig. 6 - Registro típico de um ensaio entre furos utilizando a fonte sísmica descrita em conjunto com um sensor triortogonal (V componente vertical; T - componente transversal). Taioli, 1999.

Fig. 6 - Typical result of a crosshole test using the source described and a triortogonal sensor ( $V$ - vertical component; $T$ - transversal component). Taioli, 1999. 
fontes sísmicas convencionais (comerciais), se as condições de acoplamento mecânico entre revestimento e maciço não são adequadas, o sinal recebido no sensor não permite uma interpretação confiável, independentemente da quantidade de energia gerada pela fonte e pelo número de empilhamentos adotado. Portanto, a experiência tem mostrado ser muito mais importante a boa execução do furo de sondagem, instalação do revestimento e preenchimento do espaço anelar com argamassa, do que a quantidade de energia gerada pela fonte.

Portanto, os testes efetuados procuraram analisar tais características em terrenos com diferentes coeficientes de atenuação, em situações reais de aplicação do método. Tais testes evidenciaram que esta fonte sísmica é perfeitamente aplicável aos trabalhos de engenharia de fundações, atendidas as condições de acoplamento entre o revestimento e o maciço.

A figura 5 mostra um registro típico da componente vertical com inversão de polaridade de excitação, permitindo identificar claramente a chegada da onda $\mathrm{S}$ e medir com grande precisão e de forma extremamente simples.

Em adição, caso se utilize sensor triortogonal, não é necessária fonte adicional de ondas P. Isto se deve ao fato de que a fonte gera, concomitantemente às ondas $\mathrm{S}$, ondas $\mathrm{P}$, que são captadas pelos sensores horizontais que, na pior das hipóteses, estarão inclinados $45^{\circ}$ em relação à fonte. A figura 6 apresenta um registro obtido com sensor triortogonal, sendo possível observar as chegadas da onda $\mathrm{P}$ (primeiras chegadas) e da onda $\mathrm{S}$ (com maior amplitude e menor freqüência); nesta figura fica claro que o tempo de chegada da onda $\mathrm{P}$ deve ser obtido dos registros das componentes transversais, enquanto o registro da componente vertical oferece melhor resolução da onda $\mathrm{S}$.

\section{CONCLUSÕES}

Foram discutidos alguns aspectos do ensaio entre furos ("crosshole") com objetivos de estudos geotécnicos e suas limitações considerando a prática da engenharia geotécnica do Brasil. Um dos motivos que fazem com que tal técnica seja tão pouco utilizada é, sem dúvida, o custo envolvido na realização do ensaio. Este trabalho apresenta um modelo de fonte sísmica apropriada para ensaios entre furos, capaz de gerar ondas $\mathrm{S}$ com inversão de polaridade, cujo principal diferencial de outras já existentes é seu tamanho. O diâmetro conseguido com o desenho proposto possibilitou sua aplicação em furos de sondagem percussiva utilizados rotineiramente em investigação geotécnica para fundações em solos. Tal diferença, apesar de sutil, permite a execução dos ensaios sem que seja necessária a perfuração de furos complementares o que, em si, já proporciona importante decréscimo no custo final do ensaio. Testes realizados em situações normais de campo evidenciaram que a energia gerada é suficiente para a execução dos ensaios com os equipamentos sismográficos atuais. Em adição, devido a seu pequeno tamanho e peso mostrou-se ser de fácil instalação e apropriada operacionalidade.

\section{AGRADECIMENTOS}

À Fundação de Amparo à Pesquisa do Estado de São Paulo-FAPESP, processo 96/2225-2 e ao Conselho Nacional de Pesquisas - CNPq, processo 300.364/96-2, que possibilitaram a elaboração deste trabalho. Aos revisores anônimos pelas valiosas críticas e sugestões.

\section{REFERÊNCIAS}

ASTM - D4428/D4428M-84., 1990. Standard test methods for crosshole seismic testing, In Annual Book of ASTM Standards, Sec.4, Construction, v.04-08, Soil and Rock; Dimension Stone; Geosynthetics, 674-688.

Barros, J.M.C., 1997. Módulo de cisalhamento dinâmico de solos tropicais. São Paulo. (Tese de Doutoramento Escola Politécnica da Universidade de São Paulo), 637pp.

Borm, G.W., 1978. Methods from exploration seismology: Reflection, Refraction and Borehole Prospecting. Proceedings of the Symposium on Dynamical Methods in Soil and Rock Mechanics. V.3, Rock Dynamics and Geophysical Aspects, Balkema, Rotterdam.

Butler, D.K.\& Curro, J.R., 1981. Crosshole seismic testing - Procedures and pitfalls. Geophysics, 47: 23-29. 
Dourado, J.C., 1984. A utilização da sísmica na determinação de parâmetros elásticos de maciços rochosos e terrosos “in situ”. São Paulo, ABGE, (Publicação Especial), 12 pp.

Dourado, J.C., Prado, R.L., Taioli, F., Iyomasa, W.S. \& Cordeiro, R.P., 1994. O ensaio crosshole e as correlações com outros ensaios geotécnicos. 10 Cong. Bras. Mec. Solos e Eng. Fund., 2: 523-529, Foz do Iguaçu, PR. g. Denver, 1977. ASTM. Special Technical Publication 654. p. 3-29.

Elbring, G.L., Hardee, H.C. \& Paulsson, B.N.P., 1989. A test of a controlled downhole seismic source. Geophysics, 54: 1193-1198.

Gucunski, N. \& Woods, R.D., 1991. Instrumentation for SASW testing. ASCE. Recent Advances in Instrumentation, Data Acquisition and Testing in Soil Dynamics, (Geotechnical Special Publication No 29), 1-16.

Hoar, R.J. \& Stokoe II, K.H., 1978. Generation and measurement of shear waves "in situ". Symposium on dynamic geotechnical testing. Denver, 1977. ASTM (Special Technical Publication 654) ASTM, 3-29.

Jung, Y., Ibrahim, A. \& Borns, D., 1991. Mapping fracture zones in salt: High-resolution, cross-gallery seismic tomography: TheLeading Edge, 10: 37 39.

McLamore, V.R., Anderson, D.G. \& Espana, C., 1978. Crosshole testing using explosive and mechanical energy sources. In: Symposium
Dynamic Geotechnical Testing, Colorado, 1977. ASTM (Special Technical Publication 654), 3055.

Prado, R.L., 1994. O ensaio sísmico entre furos ("crosshole") no estudo de maciços terrosos e rochosos. São Paulo. (Dissertação de Mestrado, Instituto Astronômico e Geofísico da Universidade de São Paulo), 123 pp.

Rector III, J.W., 1995. Crosswell methods: where are we, where are we going?. Geophysics, 60: 629-630.

Sassa, K., 1988. Suggested methods for seismic testing within and between boreholes. International Journal of Rock Mechanics and Mining Sciences \& Geomechanics Abstracts, 25: 447-472

Sobral, A.C.S., Prado, R.L. \& Rocha, H.C., 1993. Perspectivas de aplicação do ensaio sísmico "cross-hole" na determinação de parâmetros geotécnicos. Anais 7 Cong. Bras. Geol. Eng., 1: 227-240, Poços de Caldas, MG.

Stokoe II, K.H. \& Woods, R.D., 1972. In situ shear wave velocity by crosshole method. J. Soil Mech. and Found. Division, 98: 443-460.

Stokoe II, K.H., Wright, S.G., Bay, J.A. \& Roesset, J.M., 1994. Characterization of Geotechnical Sites by SASW Method. In: ISSMFE Technical Committee 10, Woods, R.D., ed. New Delhi, Oxford Publishers. p. 15-25.

Taioli, F., 1999. Avaliação de propriedades elásticas dinâmicas de materiais geológicos da RMSP. (Tese de Livre Docência, Instituto de Geociências da Universidade de São Paulo) São Paulo 87 pp.

\section{SMALL SIZE SHEAR WAVE SOURCE FOR CROSSHOLE TESTS}

There are three main methods to determine the propagation velocities of the in situ $\mathrm{P}$ and $\mathrm{S}$ waves of the different strata and, consequently, to calculate the dynamic elastic parameters of the rock or soil massifs. They are the crosshole, the seismic refraction using both longitudinal and transversal geophones and the SASW (Spectral Analysis of Surface Waves).

While the two first allow the determination of the $\mathrm{P}$ and $\mathrm{S}$ wave propagation velocities, and the derivation of elastic moduli, the last only allows the determination of the strata $\mathrm{S}$ wave velocity, from which the rigidity modulus $(\mathbf{G})$ can be derived. In addition, while the refraction and SASW methods analyze the wave propagation velocities from the surface, the crosshole test is carried out downhole minimizing the attenuation and other interferences due to underlying strata.

The application of the crosshole method implies in an execution cost which is composed by the cost of the boreholes drilling, casing installation and the filling of the annular space with cement mortar, besides the test cost itself. Otherwise, the investigation phase during a foundation design is based mostly on SPT (Standard Penetration Tests) that includes the execution of a borehole. This 
borehole is normally abandoned after its completion even when further studies are necessary.

To decrease the cost of crosshole test and primarily to suit the instrumentation to the usual market practice, a low-cost, small size seismic source for crosshole test was developed.

The seismic source was designed and developed to be installed in $75 \mathrm{~mm}(\sim 3 ")$ diameter boreholes, with PVC casing with an external diameter of $50 \mathrm{~mm}(\sim 2 ")$. Note that these dimensions allow both the casing and source to be installed in the investigation (SPT) boreholes. The source consists of a movable piston with two shocker ends, which slide on two guide-tubes when moved by a steel cable from the surface. The body of the source is fixed to the walls of the borehole by means of a pneumatic system. This allows generating an upward shear wave (when the steel cable is pulled) or a downward shear wave (when the steel cable is released). The comparison of both results (upwards and downwards) will clearly show the inversion of polarity, which means the exact instant of the S wave's arrival.

The trigger system consists of a piezoelectric ceramic installed in the superior shocker end. A shielded cable transmits the signal generated by the ceramic to the seismograph. The positive signal is taken from the superior surface of the ceramic, while the negative signal is obtained from the chassis, once the negative pole of the ceramic is placed on the body of the source by a thin layer of adhesive epoxy.

Tests carried out in laboratory and field showed that the source generates very good amplitude of S (vertical) wave. It is important to remember that a vertical sensitive sensor placed in a parallel borehole will record the $\mathrm{S}$ wave. The tests also showed that, when using a triortogonal sensor, the P wave could be perfectly recorded, while when using a standard vertical sensor, despite the possibility of recording the $\mathrm{P}$ wave, the first break may not be precise. In addiction, tests showed that despite the relative small energy generated, a good result is much more due to the condition of the casing-borehole coupling (which is guaranteed by the perfect filling of the annular space between them) than to the amount of energy generated by the source.

Finally, the source presented in this paper has a similar design of others commercially available. Otherwise, its small size that allows it to be used in the same borehole made during the investigation phase for building foundation design, and, consequently, acquire important information about the elastic behavior of the materials contributes to decrease the overall cost of the study.

\section{NOTE ABOUT THE AUTHOR}

\begin{abstract}
Fabio Taioli
Formou-se em Geologia pela USP em 1973. Foi geofísico da Petrobrás até 1975 quando transferiuse para o Instituto de pesquisas Tecnológicas do Estado de são Paulo - IPT. Obteve, em 1987, seu MSc em Mining Engineering pela The Pennsylvania State University na área de Geomecânica. Doutorou-
\end{abstract}

se pelo Instituto de Geociências da USP em 1992 na área de Geofísica Aplicada. Transferiu-se para o IGUSP em 1995 onde é Professor Associado. Sua área de interesse é a aplicação de métodos geofísicos a problemas de engenharia civil e de minas e meio ambiente. É membro da SBGf, SEG, EEGS, IAEG, ISRM, ABGE, ABMS, CBMR. 